\title{
Conditional Symmetries and the Quantization of Bianchi Type I Vacuum Cosmologies with and without Cosmological Constant
}

\author{
T. Christodoulakis* T. Gakis \& G. O. Papadopoulos ${ }^{\dagger}$ \\ University of Athens, Physics Department \\ Nuclear 83 Particle Physics Section \\ Panepistimioupolis, Ilisia GR 157-71, Athens, Hellas
}

\begin{abstract}
In this work, the quantization of the most general Bianchi Type I geometry, with and without a cosmological constant, is considered. In the spirit of identifying and subsequently removing as many gauge degrees of freedom as possible, a reduction of the initial 6-dimensional configuration space is presented. This reduction is achieved by imposing as additional conditions on the wave function, the quantum version of the -linear in momenta- classical integrals of motion (conditional symmetries). The vector fields inferred from these integrals induce, through their integral curves, motions in the configuration space which can be identified to the action of the automorphism group of Type I, i.e. $G L(3, \Re)$. Thus, a wave function depending on one degree of freedom, namely the determinant of the scale factor matrix, is found.

A measure for constructing the Hilbert space is proposed. This measure respects the above mentioned symmetries, and is also invariant under the classical property of covariance under arbitrary scalings of the Hamiltonian (quadratic constraint).
\end{abstract}

*e-mail: tchris@cc.uoa.gr

†e-mail: gpapado@cc.uoa.gr 


\section{Introduction}

Since the conception by Einstein of General Relativity Theory, a great many efforts have been devoted by many scientists to the construction of a consistent quantum theory of gravity. These efforts can de divided into two main approaches:

(a) perturbative, in which one splits the metric into a background (kinematical) part and a dynamical one: $g_{\mu \nu}=\eta_{\mu \nu}+h_{\mu \nu}$ and tries to quantize $h_{\mu \nu}$. The only conclusive results existing, are that the theory thus obtained is highly nonrenormalizable [1].

(b) non perturbative, in which one tries to keep the twofold role of the metric (kinematical and dynamical) intact. A hallmark in this direction is canonical quantization.

In trying to implement this scheme for gravity, one faces the problem of quantizing a constrained system. The main steps one has to follow are:

(i) define the basic operators $\widehat{g}_{\mu \nu}$ and $\widehat{\pi}^{\mu \nu}$ and the canonical commutation relation they satisfy.

(ii) define quantum operators $\widehat{H}_{\mu}$ whose classical counterparts are the constraint functions $H_{\mu}$.

(iii) define the quantum states $\Psi[g]$ as the common null eigenvector of $\widehat{H}_{\mu}$, i.e. those satisfying $\widehat{H}_{\mu} \Psi[g]=0$. (As a consequence, one has to check that $\widehat{H}_{\mu}$, form a closed algebra under the basic Canonical Commutation Relations (CCR).)

(iv) find the states and define the inner product in the space of these states.

It is fair to say that the full program has not yet been carried out, although partial steps have been made [2]. Concerning point (iii) we deem it pertinent to clarify the meaning of the imposition of the quantum constraints upon $\Psi[g]$. A straightforward (modulo regularization prescriptions) but tedious calculation shows that any functional which is not a scalar functional of the curvature invariants (see [3]) does not solve the linear constraints. Therefore, the imposition of the linear constraints, ensures that the wave functional will be a (scalar) functional of the 3-geometry and not of the coordinate system. Then, the dynamical evolution is provided by the quadratic constraint; the consistency of the quantum algebra, guarantees that the final wave functional, will be independent of the 4 dimensional coordinate system.

In the absence of a full solution to the problem, people have turned to what is generally known as quantum cosmology. This is an approximation to quantum gravity in which one freezes out all but a finite number of degrees of freedom, and quantizes the rest. In this way one is left with a much more manageable problem that is essentially quantum mechanics with constraints. Over the years, many models have appeared in the literature [4. In most of them, the minisuperspace is flat and the gravitational field is represented by no more than three degrees of freedom (generically the three scale factors of some anisotropic Bianchi Type model [5]). 
In a series of earlier publications [6], we have considered the quantization of the most general spatially homogeneous spacetime for various Bianchi Types. Thus, our -in principle- dynamical variables were the scale factors $\gamma_{\alpha \beta}(t)$, the lapse function $N(t)$ and the shift vector $N^{\alpha}(t)$.

The presence of the linear constraints -along with the conditional symmetries of the corresponding Hamiltonian- enabled a reduction of the initial configuration space to a lower dimensional one, spanned by the curvature invariants characterizing the 3 -geometry. The ultimate justification of this reduction is the fact that -from the point of view of the 3-geometry - the omitted degrees of freedom, are not physical but gauge [7].

The case of Bianchi Type I geometries, has been repeatedly treated in the literature both at the classical level [8] and the quantum level [9]. In all these works, the scale factor matrix is taken to be diagonal. The rôle of the topology of the 3-slices, is emphasized in the work of Ashtekar and particularly in that of Hervik where a non trivial $T^{3}$ topology leads to a nine dimensional moduli space. The main reason for this plethora of treatments of Type I, is the simplicity brought by the vanishing structure constants, i.e. the high spatial symmetry of the model. It is true that at the classical level, the scale factor matrix, can be diagonalized on mass-shell -through a constant matrix [10]- while the shift can be set equal to zero. However, if one intends to give weight to all states, one has to start with the most general form which is described by the 6 scale factors $\gamma_{\alpha \beta}(t)$ and the lapse function $N(t)$. The absence of $H_{\alpha} \mathrm{s}$ due to the vanishing of the $C_{\beta \gamma}^{\alpha} \mathrm{s}$, implies that in principle all $\gamma_{\alpha \beta} \mathrm{s}$ are candidates as arguments for the wave function which solves the quadratic constraint (Wheeler-DeWitt equation). This is in contrast to what happens in other Bianchi Types [6] where, less or equal to 3, combinations of $\gamma_{\alpha \beta} \mathrm{S}$ and $C_{\beta \gamma}^{\alpha}$ s, parameterize the reduced configuration space.

In this short communication, we present a complete reduction of the initial configuration space for Bianchi Type I geometry -by extracting as many gauge degrees of freedom, as possible. Two separate cases are considered; when the cosmological constant is present and when is not. In either case, a wave function which depends on one degree of freedom (namely the determinant of the scale factor matrix) is found, by imposing on it, the quantum versions of all classical integrals of motion as additional conditions.

\section{Bianchi Type I, the $\Lambda \neq 0$ case}

In this work, we will quantize the known action corresponding to the most general Bianchi Type I cosmologies, i.e. the action giving Einstein's Field Equations derived from the line element:

$$
d s^{2}=\left(-N^{2}(t)+N_{\alpha}(t) N^{\alpha}(t)\right) d t^{2}+2 N_{\alpha}(t) \sigma_{i}^{\alpha}(x) d x^{i} d t+\gamma_{\alpha \beta}(t) \sigma_{i}^{\alpha}(x) \sigma_{j}^{\beta}(x) d x^{i} d x^{j}
$$

where $\sigma_{i}^{\alpha}$ are the invariant basis one-forms of the homogeneous surfaces of simultaneity $\Sigma_{t}$, satisfying:

$$
d \sigma^{\alpha}=C_{\beta \gamma}^{\alpha} \sigma^{\beta} \wedge \sigma^{\gamma} \Leftrightarrow \sigma_{i, j}^{\alpha}-\sigma_{j, i}^{\alpha}=2 C_{\beta \gamma}^{\alpha} \sigma_{i}^{\gamma} \sigma_{j}^{\beta}
$$


with $C_{\beta \gamma}^{\alpha}$ being the structure constants of the corresponding isometry group. In 3 dimensions, the tensor $C_{\beta \gamma}^{\alpha}$ admits a unique decomposition in terms of a contravariant symmetric tensor density of weight $-1, m^{\alpha \beta}$, and a covariant vector $\nu_{\alpha}=\frac{1}{2} C_{\alpha \rho}^{\rho}$ as follows:

$$
C_{\beta \gamma}^{\alpha}=m^{\alpha \delta} \varepsilon_{\delta \beta \gamma}+\nu_{\beta} \delta_{\gamma}^{\alpha}-\nu_{\gamma} \delta_{\beta}^{\alpha}
$$

For the Class A $\left(\nu_{\alpha}=0\right)$ Bianchi Type I, this matrix is [1]:

$$
m^{\alpha \beta}=\left(\begin{array}{ccc}
0 & 0 & 0 \\
0 & 0 & 0 \\
0 & 0 & 0
\end{array}\right)
$$

resulting in vanishing structure constants.

As is well known [12], the Hamiltonian of the above system is $H=\widetilde{N}(t) H_{0}+N^{\alpha}(t) H_{\alpha}$, where:

$$
H_{0}=\frac{1}{2} L_{\alpha \beta \mu \nu} \pi^{\alpha \beta} \pi^{\mu \nu}+\gamma R+\gamma \Lambda
$$

is the quadratic constraint, with:

$$
\begin{aligned}
& L_{\alpha \beta \mu \nu}=\gamma_{\alpha \mu} \gamma_{\beta \nu}+\gamma_{\alpha \nu} \gamma_{\beta \mu}-\gamma_{\alpha \beta} \gamma_{\mu \nu} \\
& R=C_{\lambda \mu}^{\beta} C_{\theta \tau}^{\alpha} \gamma_{\alpha \beta} \gamma^{\theta \lambda} \gamma^{\tau \mu}+2 C_{\beta \delta}^{\alpha} C_{\nu \alpha}^{\delta} \gamma^{\beta \nu}+4 C_{\mu \nu}^{\mu} C_{\beta \lambda}^{\beta} \gamma^{\nu \lambda}
\end{aligned}
$$

$\gamma$ being the determinant of $\gamma_{\alpha \beta}$, and:

$$
H_{\alpha}=C_{\alpha \rho}^{\mu} \gamma_{\beta \mu} \pi^{\beta \rho}
$$

are the linear constraints. Note that $\widetilde{N}$ appearing in the Hamiltonian, is to be identified with $N / \sqrt{\gamma}$. For all Class A Types, the canonical equations of motion, following from (2.5), are equivalent to Einstein's equations derived from line element (2.1) - see [12].

The quantities $H_{0}, H_{\alpha}$ are weakly vanishing [13], i.e. $H_{0} \approx 0, H_{\alpha} \approx 0$. For all Class A Bianchi Types $\left(C_{\alpha \beta}^{\alpha}=0\right)$, it can be seen - using the basic PBR $\left\{\gamma_{\alpha \beta}, \pi^{\mu \nu}\right\}=\delta_{\alpha \beta}^{\mu \nu}$ - that these constraints are first class, obeying the following algebra

$$
\begin{aligned}
& \left\{H_{0}, H_{\alpha}\right\}=0 \\
& \left\{H_{\alpha}, H_{\beta}\right\}=-\frac{1}{2} C_{\alpha \beta}^{\gamma} H_{\gamma},
\end{aligned}
$$

which ensures their preservation in time, i.e. $\dot{H}_{0} \approx 0, \dot{H}_{\alpha} \approx 0$, and establishes the consistency of the action.

If we follow Dirac's general proposal [13 for quantizing this action, we have to turn $H_{0}, H_{\alpha}$, into operators annihilating the wave function $\Psi$.

In the Schrödinger representation:

$$
\begin{aligned}
& \gamma_{\alpha \beta} \rightarrow \widehat{\gamma}_{\alpha \beta}=\gamma_{\alpha \beta} \\
& \pi^{\alpha \beta} \rightarrow \widehat{\pi}^{\alpha \beta}=-i \frac{\partial}{\partial \gamma_{\alpha \beta}}
\end{aligned}
$$


with the relevant operators satisfying the basic Canonical Commutation Relations (CCR) -corresponding to the classical ones:

$$
\left[\widehat{\gamma}_{\alpha \beta}, \widehat{\pi}^{\mu \nu}\right]=i \delta_{\alpha \beta}^{\mu \nu}=\frac{i}{2}\left(\delta_{\alpha}^{\mu} \delta_{\beta}^{\nu}+\delta_{\beta}^{\mu} \delta_{\alpha}^{\nu}\right)
$$

In Bianchi Type I $\left(C_{\beta \gamma}^{\alpha}=0\right)$, the second of (2.6) gives $R=0$, while relations (2.7) vanish identically, and the algebra (2.8) is trivially satisfied. Thus, (2.5), reads:

$$
H_{0}=\frac{1}{2} L_{\alpha \beta \mu \nu} \pi^{\alpha \beta} \pi^{\mu \nu}+\gamma \Lambda
$$

Thus, the only operator which must annihilate the wave function, is $\widehat{H}_{0}$; and the Wheeler-DeWitt equation $\widehat{H}_{0} \Psi=0$, will produce a wave function, initially residing on a 6 -dimensional configuration space -spanned by $\gamma_{\alpha \beta}$ s. The discussion however, does not end here. If the linear constraints existed, a first reduction of the initial configuration space, would take place [14]. New variables, instead of the 6 scale factors, would emerge -say $q^{i}$, with $i<6$. Then a new "physical" metric would be induced:

$$
g^{i j}=L_{\alpha \beta \mu \nu} \frac{\partial q^{i}}{\partial \gamma_{\alpha \beta}} \frac{\partial q^{j}}{\partial \gamma_{\mu \nu}}
$$

According to Kuchař's and Hajicek's [14] prescription, the "kinetic" part of $H_{0}$ would have to be realized as the conformal Laplacian (in order for the equation to respect the conformal covariance of the classical action), based on the physical metric (2.12). In the presence of conditional symmetries, further reduction can take place, a new physical metric would then be defined similarly, and the above mentioned prescription, would have to be used after the final reduction [6, 15].

The case of Bianchi Type I, is an extreme example in which all the linear constraints, vanish identically; thus no initial physical metric, exists -another peculiarity reflecting the high spatial symmetry of the model under consideration. In compensation, a lot of integrals of motion exist ant the problem of reduction, finds its solution through the notion of "Conditional Symmetries". These linear in momenta integrals of motion, if seen as vector field on the configuration space spanned by $\gamma_{\alpha \beta}$ s, induce -through their integral curves- motions of the form $\widetilde{\gamma}_{\alpha \beta}=\Lambda_{\alpha}^{\mu} \Lambda_{\beta}^{\nu} \gamma_{\mu \nu}, \Lambda \in G L(3, \Re)$ (see section 2 of [7]) which not only are identical to the action of spatial diffeomorphisms, but also describe the action of the automorphism group - since $G L(3, \Re)$ is the Aut $(\mathrm{G})$ for Type I [16].

The generators of this automorphism group, are (in a collective form and matrix notation) the following 9 -one for each parameter:

$$
\lambda_{(I) \beta}^{\alpha}=\left(\begin{array}{ccc}
a & \beta & \delta \\
\epsilon & \zeta & \eta \\
\theta & \sigma & \rho
\end{array}\right), \quad I \in[1, \ldots, 9]
$$

with the defining property:

$$
C_{\mu \nu}^{\alpha} \lambda_{\alpha}^{\kappa}=C_{\mu \sigma}^{\kappa} \lambda_{\nu}^{\sigma}+C_{\sigma \nu}^{\kappa} \lambda_{\mu}^{\sigma}
$$


Exponentiating all these matrices, one obtains the outer automorphism group of Type I, since there is not Inner Automorphism subgroup (all structure constants vanish).

For full pure gravity, Kuchař [15] has shown that there are no other first-class functions, homogeneous and linear in the momenta, except the linear constraints. If however, we impose extra symmetries (e.g. the Bianchi Type I -here considered), such quantities may emerge -as it will be shown. We are therefore -according to Dirac [13]- justified to seek the generators of these extra symmetries; their quantum-operator analogues will be imposed as additional conditions on the wave function. The justification for such an action, is obvious since these generators correspond to spatial diffeomorphisms -which are the covariance of the theory. Thus, these additional conditions are expected to lead us to the final reduction, by revealing the true degrees of freedom. Such quantities are, generally, called in the literature "Conditional Symmetries" [15.

From matrices (2.13), we can construct the linear -in momenta- quantities:

$$
E_{(I)}=\lambda_{(I) \beta}^{\alpha} \gamma_{\alpha \rho} \pi^{\rho \beta}
$$

In order to write analytically these quantities, the following base is chosen:

$$
\begin{aligned}
& \lambda_{1}=\left(\begin{array}{lll}
0 & 1 & 0 \\
0 & 0 & 0 \\
0 & 0 & 0
\end{array}\right), \quad \lambda_{2}=\left(\begin{array}{ccc}
0 & 0 & 1 \\
0 & 0 & 0 \\
0 & 0 & 0
\end{array}\right), \quad \lambda_{3}=\left(\begin{array}{lll}
0 & 0 & 0 \\
0 & 0 & 1 \\
0 & 0 & 0
\end{array}\right) \\
& \lambda_{4}=\left(\begin{array}{lll}
0 & 0 & 0 \\
0 & 0 & 0 \\
0 & 1 & 0
\end{array}\right), \quad \lambda_{5}=\left(\begin{array}{ccc}
0 & 0 & 0 \\
0 & 0 & 0 \\
1 & 0 & 0
\end{array}\right), \quad \lambda_{6}=\left(\begin{array}{lll}
0 & 0 & 0 \\
1 & 0 & 0 \\
0 & 0 & 0
\end{array}\right) \\
& \lambda_{7}=\left(\begin{array}{ccc}
1 & 0 & 0 \\
0 & -1 & 0 \\
0 & 0 & 0
\end{array}\right), \quad \lambda_{8}=\left(\begin{array}{ccc}
0 & 0 & 0 \\
0 & -1 & 0 \\
0 & 0 & 1
\end{array}\right), \quad \lambda_{9}=\left(\begin{array}{lll}
1 & 0 & 0 \\
0 & 1 & 0 \\
0 & 0 & 1
\end{array}\right)
\end{aligned}
$$

It is straightforward to calculate the Poisson Brackets between $E_{(I)}$ and $H_{0}$ :

$$
\left\{E_{(I)}, H_{0}\right\}=-\gamma \Lambda \lambda_{a}^{a}
$$

But, it holds that:

$$
\dot{E}_{(I)}=\left\{E_{(I)}, H_{0}\right\}=-\gamma \Lambda \lambda_{a}^{a}
$$

-the last equality emerging by virtue of (2.17). Thus:

$$
\dot{E}_{(I)}=\left\{E_{(I)}, H_{0}\right\}=0 \Rightarrow E_{(I)}=K_{(I)}=\text { constants, } \quad I \in[1, \ldots, 8]
$$

We therefore conclude that, the first eight quantities $E_{(I)}$, are first-class, and thus integrals of motion. Out of the eight quantities $E_{(I)}$, only five are functionally independent (i.e. linearly independent, if we allow for the coefficients of the linear combination, to be functions of the $\gamma_{\alpha \beta} \mathrm{s}$ ); numerically, they are all independent.

The algebra of $E_{(I)}$ can be easily seen to be:

$$
\left\{E_{(I)}, E_{(J)}\right\}=-\frac{1}{2} C_{I J}^{M} E_{(M)}, \quad I, J, M \in[1, \ldots, 9]
$$


where:

$$
\left[\lambda_{(I)}, \lambda_{(J)}\right]=C_{I J}^{M} \lambda_{(M)}, \quad I, J, M \in[1, \ldots, 9]
$$

the square brackets denoting matrix commutation.

The non vanishing structure constants of the algebra (2.21), are found to be:

$$
\begin{array}{lllll}
C_{13}^{2}=1 & C_{15}^{4}=-1 & C_{16}^{7}=1 & C_{18}^{1}=-1 & C_{17}^{1}=-2 \\
C_{24}^{1}=1 & C_{25}^{7}=1 & C_{25}^{8}=-1 & C_{26}^{3}=-1 & C_{27}^{2}=-1 \\
C_{28}^{2}=1 & C_{34}^{8}=-1 & C_{35}^{6}=1 & C_{37}^{3}=1 & C_{38}^{3}=2 \\
C_{46}^{5}=1 & C_{47}^{4}=-1 & C_{48}^{4}=-2 & C_{57}^{5}=1 & C_{58}^{5}=-1 \\
C_{67}^{6}=2 & C_{68}^{6}=1 & & &
\end{array}
$$

At this point, in order to achieve the desired reduction, we propose that the quantities $E_{(I)}$-with $I \in[1, \ldots, 8]$ - must be promoted to operational conditions acting on the requested wave function $\Psi$-since they are first class quantities and thus integrals of motion (see (2.19)). In the Schrödinger representation:

$$
\widehat{E}_{(I)} \Psi=-i \lambda_{(I) \alpha}^{\tau} \gamma_{\tau \beta} \frac{\partial \Psi}{\partial \gamma_{\alpha \beta}}=K_{(I)} \Psi, \quad I \in[1, \ldots, 8]
$$

In general, systems of equations of this type, must satisfy consistency conditions decreed by the Frobenius Theorem:

$$
\begin{aligned}
& \widehat{E}_{(J)} \Psi=K_{(J)} \Psi \Rightarrow \widehat{E}_{(I)} \widehat{E}_{(I)} \Psi=K_{(I)} K_{(J)} \Psi \\
& \widehat{E}_{(I)} \Psi=K_{(I)} \Psi \Rightarrow \widehat{E}_{(J)} \widehat{E}_{(I)} \Psi=K_{(J)} K_{(I)} \Psi
\end{aligned}
$$

Subtraction of these two and usage of (2.20), results in:

$$
K_{I J}^{M} \widehat{E}_{(M)} \Psi=0 \Rightarrow C_{I J}^{M} K_{(M)}=0
$$

This relation constitutes a selection rule for the numerical values of the integrals of motion. In view of the Lie Algebra (2.22), selection rule (2.25) imposes that all $K \mathrm{~s}$ vanish, i.e. $K_{1}=\ldots=K_{8}=0$. This fact restores the action of the diffeomorphisms as covariances of the quantum theory, in the sense that now, we have conditions of the form $\widehat{E}_{(I)} \Psi=0$. Instead, if we also had $E_{(9)}$ (as is the case $\Lambda=0$ ) then $K_{9}$ would remain arbitrary. With this outcome, and using the method of characteristics [17], the system of the five functionally independent P.D.E. s (2.23), can be integrated. The result is:

$$
\Psi=\Psi(\gamma)
$$

i.e. an arbitrary (but well behaved) function of $\gamma$-the determinant of the scale factor matrix.

A note is pertinent here; from basic abstract algebra, is well known that the basis of a linear vector space, is unique -modulo linear mixtures. Thus, although the form of the system (2.23) is base dependent, its solution (2.26), is base independent. 
The next step, is to construct the Wheeler-DeWitt equation which is to be solved by the wave function (2.26). The degree of freedom, is 1 ; the $q=\gamma$. According to Kuchař's proposal [14], upon quantization, the kinetic part of Hamiltonian (2.11) is to be realized as the conformal Beltrami operator - based on the induced physical metric -according to (2.12), with $q=\gamma$ :

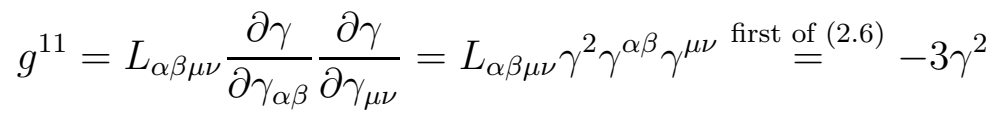

In the Schrödinger representation:

$$
\frac{1}{2} L_{\alpha \beta \mu \nu} \pi^{\alpha \beta} \pi^{\mu \nu} \rightarrow-\frac{1}{2} \square_{c}^{2}
$$

where:

$$
\square_{c}^{2}=\square^{2}=\frac{1}{\sqrt{g_{11}}} \partial_{\gamma}\left\{\sqrt{g_{11}} g^{11} \partial_{\gamma}\right\}
$$

is the 1 -dimensional Laplacian based on $g_{11}\left(g^{11} g_{11}=1\right)$. Note that in 1 -dimension the conformal group is totally contained in the G.C.T. group, in the sense that any conformal transformation of the metric can not produce any change in the -trivial-geometry and is thus reachable by some G.C.T. Therefore, no extra term in needed in (2.29), as it can also formally be seen by taking the limit $d=1, R=0$ in the general definition:

$$
\square_{c}^{2} \equiv \square^{2}+\frac{(d-2)}{4(d-1)} R=\square^{2}
$$

Thus:

$$
H_{0} \rightarrow \widehat{H}_{0}=-\frac{1}{2}\left(-3 \gamma^{2} \frac{\partial^{2}}{\partial \gamma}-3 \gamma \frac{\partial}{\partial \gamma}\right)+\Lambda \gamma
$$

So, the Wheeler-DeWitt equation -by virtue of (2.26)-, reads:

$$
\widehat{H}_{0} \Psi=\gamma^{2} \Psi^{\prime \prime}+\gamma \Psi^{\prime}+\frac{2}{3} \gamma \Lambda \Psi=0
$$

The general solution to this equation, is:

$$
\Psi(\gamma)=c_{1} J_{0}\left(2 \sqrt{\frac{2 \gamma \Lambda}{3}}\right)+c_{2} Y_{0}\left(2 \sqrt{\frac{2 \gamma \Lambda}{3}}\right)
$$

where $J_{n}$ and $Y_{n}$, are the Bessel Functions of the first and second kind respectively -both with zero argument- and $c_{1}, c_{2}$, arbitrary constants.

Some comments on this wave function. Indeed, at first sight, the fact that $\Psi$ depends only on one argument and particularly on $\gamma$, seems to point to some undesirable degeneracy regarding anisotropy; classically $\gamma$ can be gauged to $e^{t}$ and thus it seems as though 
the anisotropy parameter does not enter $\Psi$ at all. If, however, we reflect thoroughly, we will realize that this objection rests strongly on a -not generally accepted- mingling of the classical notion of anisotropy and the interpretation of the wave function. Indeed if we adopt the interpretation that the wave function $\Psi$ (along with a suitable measure), is to give weight to all configurations parameterized by $\gamma_{\alpha \beta}$, then the anisotropic configuration will, in general, acquire different probabilities. The degeneracy occurs only between two different anisotropic configurations with the same determinant $\gamma$. In compensation the scheme proposed here, avoids the gauge degrees of freedom as much as possible. The final probabilistic interpretation must await the selection of a proper measure, a theme which will be discussed in section 4 .

\section{$3 \quad$ Bianchi Type I, the $\Lambda=0$ case}

This section mimics the previous one, with minor changes. Indeed, if the cosmological constant $\Lambda$ is zero, some changes will take place.

The first concerns the obvious alteration to the Hamiltonian (2.11), which now reads:

$$
H_{0}=\frac{1}{2} L_{\alpha \beta \mu \nu} \pi^{\alpha \beta} \pi^{\mu \nu}
$$

This consequently, causes an alteration to the Poisson Bracket (2.17), which takes the form:

$$
\left\{E_{(I)}, H_{0}\right\}=0, \quad I \in[1, \ldots, 9]
$$

while, (2.20) still holds. Thus in the present case, there are nine integrals of motion -instead of eight. Also, the P.D.E. system (2.23) consists of nine members (instead of eight), but now out of the nine quantities $E_{(I)}$, only six are functionally independent; the previous five, plus the $E_{(9)}$. Again, using the method of characteristics [17], the system of the six functionally independent P.D.E. s (2.23), can be integrated. The result is:

$$
\Psi=c_{1} \gamma^{i K_{9} / 3}
$$

where $\gamma$ is the determinant of the scale factor and $K_{9}$, the remaining constant -according to selection rule (2.25).

The fact that this wave function does not depend on any combination of $\gamma_{\alpha \beta} \mathrm{s}$ in an arbitrary manner (i.e. $\Psi$ is not an arbitrary function of $\gamma_{\alpha \beta} \mathrm{s}$ ), might be taken as an indication that no reduced Wheeler-DeWitt equation can be written. On the other hand, this wave function does contain an arbitrary (essential) constant, which ought to be fixed by the dynamics. The puzzle can be solved by the following compromise; the initial configuration space, should be the mini-superspace i.e. we should write the Wheeler-DeWitt equation, based on the supermetric $L^{\alpha \beta \mu \nu}$.

In the Schrödinger representation:

$$
\frac{1}{2} L_{\alpha \beta \mu \nu} \pi^{\alpha \beta} \pi^{\mu \nu} \rightarrow-\frac{1}{2} \square_{c}^{2}
$$


Thus using (A.9), (A.11), A.12) for $d=3$ and $\mathcal{D}=6$, one may find respectively -see appendix:

$$
\begin{gathered}
R=\frac{15}{2} \\
L_{\alpha \beta \mu \nu} \Gamma_{\kappa \lambda}^{\alpha \beta \mu \nu}=-3 \gamma_{\kappa \lambda}
\end{gathered}
$$

and:

$$
\square_{c}^{2}=L_{\alpha \beta \mu \nu} \frac{\partial}{\partial \gamma_{\alpha \beta} \gamma_{\mu \nu}}+3 \gamma_{\kappa \lambda} \frac{\partial}{\partial \gamma_{\kappa \lambda}}+\frac{3}{2}
$$

Then Kuchař's proposal for (3.1) reads:

$$
H_{0} \rightarrow \widehat{H}_{0}=-\frac{1}{2}\left(L_{\alpha \beta \mu \nu} \frac{\partial}{\partial \gamma_{\alpha \beta} \gamma_{\mu \nu}}+3 \gamma_{\kappa \lambda} \frac{\partial}{\partial \gamma_{\kappa \lambda}}+\frac{3}{2}\right)
$$

Substitution of the wave function (3.3) in the Wheeler-DeWitt equation $\widehat{H}_{0} \Psi=0$, with $\widehat{H}_{0}$ given by the previous relation, determines the constant $K_{9}$. The outcome is:

$$
\Psi=c_{1} \gamma^{\sqrt{2} / 2}+c_{2} \gamma^{-\sqrt{2} / 2}
$$

The constants $c_{1}, c_{2}$, remain arbitrary and may be fixed after the selection of a proper measure via normalizability requirements.

\section{Measure and Probabilistic Interpretation}

In general, the issue of the selection of a measure, is an open question. For us, an important element for selecting it, is the conformal covariance; the supermetric $L^{\alpha \beta \mu \nu}$ is known only up to rescalings, because instead of $\widetilde{N}(t)$ one can take any $\bar{N}(t)=\widetilde{N}(t) e^{-2 \omega}$ (with $\omega=\omega\left(\gamma_{\alpha \beta}\right)$ ) and consequently $\bar{L}^{\alpha \beta \mu \nu}(t)=L^{\alpha \beta \mu \nu}(t) e^{2 \omega}$. This property, is also inherited to the physical metric (2.12) and is the reason for the Kuchař's recipe, adopted in this work.

It is therefore mandatory for the proposed measure $\mu$, to be such that the probability density $\mu|\Psi|^{2}$, be invariant under these scalings. Recalling that $\bar{\Psi}=\Psi e^{(2-\mathcal{D}) \omega / 2}$, we conclude that $\mu$ must scale as $\bar{\mu}=\mu e^{(\mathcal{D}-2) \omega}$.

It is not difficult to imagine such a quantity: any product of $E_{(I) \alpha \beta}$ with $E_{(J) \mu \nu}$ (where $E_{(I) \alpha \beta}=1 / 2\left(\lambda_{a}^{\kappa} \gamma_{\kappa \beta}+(\alpha \leftrightarrow \beta)\right)$ are the components of the vector field inferred from the integrals of motion $\left.E_{(I)}\right)$ has the desired property. Indeed the $E_{(I)}$ s do not scale at all, while the supermetric scales as mentioned before. The group metric $\Theta_{I J}=C_{I S}^{F} C_{J F}^{S}$ can serve to close the group indices of $E_{(I) \alpha \beta}$. So, we arrive at the quantity:

$$
\xi=L^{\alpha \beta \mu \nu} \Theta^{I J} E_{(I) \alpha \beta} E_{(J) \mu \nu}
$$


(where $\Theta^{I J}$ is the inverse of the group metric). The quantity $\xi$ transforms as: $\bar{\xi}=\xi e^{2 \omega}$. Thus we only need to take $\mu=\xi^{(\mathcal{D}-2) / 2}$ Using the Lie algebra (2.22), one obtains:

$$
\Theta_{I J} \equiv C_{I S}^{F} C_{J F}^{S}=\left(\begin{array}{ccccccccc}
0 & 0 & 0 & 0 & 0 & 6 & 0 & 0 & 0 \\
0 & 0 & 0 & 0 & 6 & 0 & 0 & 0 & 0 \\
0 & 0 & 0 & 6 & 0 & 0 & 0 & 0 & 0 \\
0 & 0 & 6 & 0 & 0 & 0 & 0 & 0 & 0 \\
0 & 6 & 0 & 0 & 0 & 0 & 0 & 0 & 0 \\
6 & 0 & 0 & 0 & 0 & 0 & 0 & 0 & 0 \\
0 & 0 & 0 & 0 & 0 & 0 & 6 & 12 & 0 \\
0 & 0 & 0 & 0 & 0 & 0 & 12 & 6 & 0 \\
0 & 0 & 0 & 0 & 0 & 0 & 0 & 0 & 0
\end{array}\right)
$$

Thus, in order to have an inverse, we restrict ourselves to the non trivial $8 \times 8$ subspace, and have:

$$
\Theta^{I J}=\left(\begin{array}{cccccccc}
0 & 0 & 0 & 0 & 0 & 1 / 6 & 0 & 0 \\
0 & 0 & 0 & 0 & 1 / 6 & 0 & 0 & 0 \\
0 & 0 & 0 & 1 / 6 & 0 & 0 & 0 & 0 \\
0 & 0 & 1 / 6 & 0 & 0 & 0 & 0 & 0 \\
0 & 1 / 6 & 0 & 0 & 0 & 0 & 0 & 0 \\
1 / 6 & 0 & 0 & 0 & 0 & 0 & 0 & 0 \\
0 & 0 & 0 & 0 & 0 & 0 & 1 / 9 & -1 / 18 \\
0 & 0 & 0 & 0 & 0 & 0 & -1 / 18 & 1 / 9
\end{array}\right)
$$

That means that in the sum of the expression (4.1), we exclude the vector $E_{9}$. After a straightforward calculation, one finds that:

$$
\xi=\frac{5}{12}
$$

The quantity $\xi^{(\mathcal{D}-2) / 2}$ in each of the two cases (i.e. with and without the cosmological constant), defines the final expression for the measure $\mu$.

A drawback of the current measure, is the loss of the hermiticity of the WheelerDeWitt operator. This however is not such a blander because, the eigenvalues of this operator, are zero and thus loss of hermiticity does not result in having complex eigenvalues. On the other hand the following welcomed features are obtained:

- In the case $\Lambda \neq 0$, the wave function which results in normalizability, is: $\Psi=c_{1} J_{0}\left(2 \sqrt{\frac{2 \gamma \Lambda}{3}}\right)$, where $J_{0}$ is the Bessel function of the first kind with zero argument. Since this function has a branch cut discontinuity in the complex z plane running from $-\infty$ to 0 , we are naturally led to consider $\Lambda$ to be positive definite -since $\gamma$ is positive definite. Also, from the behaviour of the probability density, it is inferred that models with small $\Lambda$ are much more probable. The limit $\Lambda \rightarrow 0$, gives a constant wave function and therefore constant probability. 
- In the case $\Lambda=0$, the wave function turns out to be non normalizable -if we use the measure above. This can be thought as an indication against the favour of such a model over the previous one. Of course, one could conceive of measures, e.g. gaussian ones, which would make the probability normalizable. Further investigation on this issue is irrelevant in view of the plethora of these possibilities.

\section{Discussion}

The present work is the last in a series of publications in which stress has been laid on the spirit and the usage of "Conditional Symmetries" within the programm of the Quantum Cosmology approximation towards the quantization of Gravity. It was shown how these can be used in order to achieve a reduction of the initial configuration space -a problem closely related to the symmetry of a model in general, and in particularly to the high spatial symmetry of Bianchi Type I model. Indeed, when these Conditional Symmetries are imposed on the requested wave function, i.e. the P.D.E. systems (2.23), along with their consistency conditions, a significant reduction of the configuration space takes place, by extracting as much gauge degrees of freedom, as possible. This is a sine qua non procedure in view of the fact that these conditional symmetries in Quantum Cosmology, are nothing but a part of the G.C.T. group as is explicitly shown in [7]. At the same time, most of the classical constants of motion, are set equal to zero -a fact not encountered before.

The reduced space, is one dimensional and the degree of freedom left is still gauge ( $\gamma$ is a density) if seen from the 3-slice point of view. In both of the cases under consideration, a wave function is gained and a kind of "proposed rôle" for it, is exhibited. Only in the first case, the wave function is normalizable. Also, in this case, the results are in agreement with the latest estimations, concerning $\Lambda$, i.e. that it is positive and relatively small. Another peculiarity is that in $\Lambda \neq 0$ case, there is a non zero probability when $\gamma \rightarrow 0$ but this it might be seen as a side-effect of the "preferred" coordinate system. Indeed, when $\gamma \rightarrow 0$, the same limit is attained by the determinant of the supermetric, as well. If however, one forms any metric invariant in the minisuperspace, he will see that the initially detected singularity, is apparent and is due to the choice of the coordinate system of this space, only.

At this point a remark concerning the implicit functional form of wave functions with respect to the 3 -metric, is pertinent. In the literature it is common to assume a wave function dependant upon a diagonal metric. Such an assumption is certainly understandable from the a classical point of view, but not justified quantum mechanically for two reasons:

$R_{1}$ The wave function is supposed to give weight to all states, not only to the classical ones. So, ab initio, it must depend on all 6 scale factors $\gamma_{\alpha \beta}$.

$R_{2}$ As it has been mentioned in [7, 10], there is a particular class of G.C.T.s, which preserve manifest homogeneity of the line element of the generic Bianchi Homogeneous 3-space, has a non trivial action on the configuration space spanned by 
$\gamma_{\alpha \beta}$ 's, and this action is that of the Automorphism group. Its differential description, leads to the vector fields (2.15). Classically, in some Bianchi Types (e.g. Type $V I I I, I X)$ an off mass-shell diagonalization of the scale factor matrix is possible, and in some others (e.g. Type $I$ ) the diagonalization is possible only on mass-shell [10]. If one passes to the quantum level, i.e. if one uses the generators $\lambda_{\rho}^{\alpha}$ of the corresponding simplifying automorphisms $\Lambda_{\rho}^{\alpha}$, to construct vector fields of the type $\lambda_{\rho}^{\alpha} \gamma_{\alpha \sigma} \frac{\partial}{\partial \gamma_{\rho \sigma}}$, he will encounter a very different situation; imposing these fields upon $\Psi$ depending on all $\gamma_{\alpha \beta}$ s, he will not get a $\Psi$ depending on the $\left\{\gamma_{11}, \gamma_{22}, \gamma_{33}\right\}$ only. For example the automorphism group for Bianchi Type $I X$ is $S O(3)$, so $\Lambda_{\rho}^{\alpha}$ are the rotations, and their generators, are:

$$
\lambda_{1}=\left(\begin{array}{ccc}
0 & 1 & 0 \\
-1 & 0 & 0 \\
0 & 0 & 0
\end{array}\right), \quad \lambda_{2}=\left(\begin{array}{ccc}
0 & 0 & -1 \\
0 & 0 & 0 \\
1 & 0 & 0
\end{array}\right), \quad \lambda_{3}=\left(\begin{array}{ccc}
0 & 0 & 0 \\
0 & 0 & 1 \\
0 & -1 & 0
\end{array}\right)
$$

The corresponding vector fields (2.15) upon $\Psi$ do not imply that this $\Psi$, in principle, an arbitrary function of $\left\{\gamma_{11}, \gamma_{22}, \gamma_{33}\right\}$ only -while at the classical level, the arbitrary scale factor matrix is diagonalized through the usage of three independent rotations.

On the issue of the measure, we have succeeded in constructing a measure which respects the scalings of $H_{0}$ and gives a square integrable wave function (in the $\Lambda \neq 0$ case), at the cost of loosing hermiticity of the Wheeler-DeWitt operator. The last is not a serious drawback of the whole scheme, because not only the Wheeler-DeWitt equation, constitutes a zero eigenfunction problem, but also, the eigenfunctions (i.e. the permissible wave functions) are complex functions in general - the probability density in defined as the measure times the norm of the wave function.

Lastly we would like to point out that the usage of the above techniques, is in contact with the work [18] where the strong gravity limit is treated. The connection to the strong gravity limit of all the other Bianchi Types, can be made much more explicit. The key observation is that the automorphism groups of all other Bianchi Types, are subsets of $G L(3, \Re)$ i.e. the automorphism group of Bianchi Type I. Consequently, if we adopt the point of view of imposing the appropriate subset of the linear integrals 2.15) on the wave function, corresponding to the automorphism group of the desired Bianchi Type, we will get a wave function which will satisfy the strong gravity limit of the Wheeler-DeWitt equation. For example, if we select the subset (in collective form) with:

$$
\lambda_{\beta}^{\alpha}=\left(\begin{array}{ccc}
\kappa+\mu & x & y \\
0 & \kappa & \rho \\
0 & \sigma & \mu
\end{array}\right)
$$

we will get a $\Psi$ depending on $\gamma$ and $q=\gamma_{11}^{2} / 2 \gamma$ and thus arrive at the strong gravity limit of equation (19) in the first of [6]. Likewise for all Bianchi Types.

This line of reasoning may also satisfy those which, driven by anisotropy "classical" 
considerations, would like to have more than one arguments in the wave function. Of course, our point of view is that the less gauge freedom in the wave function the better and hence we adopt the procedure all the operator analogues of the integrals of motion (2.15).

\section{Acknowledgements}

One of us (G. O. Papadopoulos) is currently a scholar of the Greek State Scholarships Foundation (I.K.Y.) and acknowledges the relevant financial support. 


\section{A Appendix}

In this appendix, we give some useful formulae, concerning the mini-superspace.

Using the results of canonical analysis in a $(d+1)$-dimensional manifold, endowed with the line element (2.1), one arrives at the notion of mini-superspace spanned by $\gamma_{\alpha \beta}$ s (co-ordinates), and having as covariant metric the following:

$$
L^{\alpha \beta \mu \nu}=\frac{1}{4}\left(\gamma^{\alpha \mu} \gamma^{\beta \nu}+\gamma^{\alpha \nu} \gamma^{\beta \mu}-2 \gamma^{\alpha \beta} \gamma^{\mu \nu}\right)
$$

while the contravariant metric, is defined as:

$$
L_{\alpha \beta \mu \nu}=\left(\gamma_{\alpha \mu} \gamma_{\beta \nu}+\gamma_{\alpha \nu} \gamma_{\beta \mu}-\frac{2}{d-1} \gamma_{\alpha \beta} \gamma_{\mu \nu}\right)
$$

in the sense that:

$$
L^{\alpha \beta \kappa \lambda} L_{\kappa \lambda \mu \nu}=\delta_{\mu \nu}^{\alpha \beta} \equiv \frac{1}{2}\left(\delta_{\mu}^{\alpha} \delta_{\nu}^{\beta}+\delta_{\nu}^{\alpha} \delta_{\mu}^{\beta}\right)
$$

The Christoffel symbols are defined as:

$$
\Gamma_{\kappa \lambda}^{\alpha \beta \mu \nu}=\frac{1}{2} L_{\kappa \lambda \rho \sigma}\left\{L^{\rho \sigma \mu \nu, \alpha \beta}+L^{\alpha \beta \rho \sigma, \mu \nu}-L^{\alpha \beta \mu \nu, \rho \sigma}\right\}
$$

where:

$$
L^{\alpha \beta \mu \nu, \rho \sigma} \equiv \frac{\partial L^{\alpha \beta \mu \nu}}{\partial \gamma_{\rho \sigma}}
$$

Combined usage of (A.1), (A.2), (A.3) and (A.4), gives:

$$
\Gamma_{\kappa \lambda}^{\alpha \beta \mu \nu}=-\frac{1}{4}\left(\gamma^{\alpha \mu} \delta_{\kappa \lambda}^{\beta \nu}+\gamma^{\alpha \nu} \delta_{\kappa \lambda}^{\beta \mu}+\gamma^{\beta \mu} \delta_{\kappa \lambda}^{\alpha \nu}+\gamma^{\beta \nu} \delta_{\kappa \lambda}^{\alpha \mu}\right)
$$

In the same spirit, the Riemann tensor is defined as follows:

$$
R_{\kappa \lambda}^{\alpha \beta \rho \sigma \mu \nu}=\Gamma_{\kappa \lambda}^{\alpha \beta \rho \sigma, \mu \nu}-\Gamma_{\kappa \lambda}^{\alpha \beta \mu \nu, \rho \sigma}+\Gamma_{\omega \xi}^{\alpha \beta \rho \sigma} \Gamma_{\kappa \lambda}^{\omega \xi \mu \nu}-\Gamma_{\omega \xi}^{\alpha \beta \mu \nu} \Gamma_{\kappa \lambda}^{\omega \xi \rho \sigma}
$$

where:

$$
\Gamma_{\kappa \lambda}^{\alpha \beta \mu \nu, \rho \sigma} \equiv \frac{\partial \Gamma_{\kappa \lambda}^{\alpha \beta \mu \nu}}{\partial \gamma_{\rho \sigma}}
$$

Contraction of $(\rho, \sigma)$ with $(\kappa, \lambda)$ results in the Ricci tensor:

$$
R^{\alpha \beta \mu \nu}=\Gamma_{\kappa \lambda}^{\alpha \beta \kappa \lambda, \mu \nu}-\Gamma_{\kappa \lambda}^{\alpha \beta \mu \nu, \kappa \lambda}+\Gamma_{\omega \xi}^{\alpha \beta \kappa \lambda} \Gamma_{\kappa \lambda}^{\omega \xi \mu \nu}-\Gamma_{\omega \xi}^{\alpha \beta \mu \nu} \Gamma_{\kappa \lambda}^{\omega \xi \kappa \lambda}
$$


A lengthy but straightforward calculation, gives:

$$
R^{\alpha \beta \mu \nu}=\frac{1}{8}\left(d \gamma^{\alpha \mu} \gamma^{\beta \nu}+d \gamma^{\alpha \nu} \gamma^{\beta \mu}-2 \gamma^{\alpha \beta} \gamma^{\mu \nu}\right)
$$

With the help of (A.8) and (A.2) the Ricci scalar is found to be:

$$
R=L_{\alpha \beta \mu \nu} R^{\alpha \beta \mu \nu}=\frac{1}{4}\left(d^{3}+d^{2}-2 d\right)
$$

Finally, the conformal Beltrami opetaror, is:

$$
\square_{c}^{2} \equiv \square^{2}+\frac{\mathcal{D}-2}{4(\mathcal{D}-1)}=L_{\alpha \beta \mu \nu} \frac{\partial^{2}}{\partial \gamma_{\alpha \beta} \gamma_{\mu \nu}}-L_{\alpha \beta \mu \nu} \Gamma_{\kappa \lambda}^{\alpha \beta \mu \nu} \frac{\partial}{\partial \gamma_{\kappa \lambda}}+\frac{\mathcal{D}-2}{4(\mathcal{D}-1)} R
$$

where $\mathcal{D}$ is the dimension of the general metric space: $\mathcal{D}=\frac{d(d+1)}{2}$, i.e. the number of the independent $\gamma_{\mu \nu}$.

One can find that:

$$
L_{\alpha \beta \mu \nu} \Gamma_{\kappa \lambda}^{\alpha \beta \mu \nu}=\frac{3-d^{2}}{d-1} \gamma_{\kappa \lambda}
$$

thus (A.10), takes the form:

$$
\square_{c}^{2} \equiv L_{\alpha \beta \mu \nu} \frac{\partial^{2}}{\partial \gamma_{\alpha \beta} \gamma_{\mu \nu}}-\frac{3-d^{2}}{d-1} \gamma_{\kappa \lambda} \frac{\partial}{\partial \gamma_{\kappa \lambda}}+\frac{\mathcal{D}-2}{4(\mathcal{D}-1)} R
$$




\section{References}

[1] M. H. Goroff and H. Sagnoti, Nuclear Physics B 266709 (1986);

G. t' Hooft, Proc. 1978 Cargese Summer School, edited by M. Levy, S. Deser, G. t' Hooft and M. Veltman, Ann. Inst. Henri Poincare 2069 (1974).

[2] T. Christodoulakis and J. Zaneli, Nuovo Cimento B 9322 (1986);

T. Christodoulakis and J. Zaneli, Classical \& Quantum Gravity 4851 (1987);

T. Christodoulakis and E. Korfiatis, Nuovo Cimento B 106239 (1991);

A. Ashtekar, Physical Review Letters 572224 (1986);

A. Ashtekar, Physical Review D 311777 (1985);

T. Jacobson and L. Smolin, Nuclear Physics B 299295 (1988);

C. Rovelli and L. Smolin, Nuclear Physics B 33180 (1990).

[3] It is known, that in 3 dimensions all metric invariants, are higher derivatives curvature invariats, while this is not true for higher dimensions:

J. Munoz Masque \& A. Valdes Morales, Journal of Physics A, 27(23) (1994)

[4] For a comprehensive list of references, see:

J. Halliwell, International Journal of Modern Physics A5 2473-2494, (1990).

[5] See for example:

P. Amsterdamski, Physical Review D 313073 (1985);

S. W. Hawking and J. C. Lutreli, Physics Letters B 14383 (1984);

S. DelCampo and A. Vilenkin, Physics Letters B 22445 (1989).

[6] T. Christodoulakis and G.O. Papadopoulos, gr-qc/0009074, Physics Letters B 501 (2001) 264-268

T. Christodoulakis G. Kofinas and G.O. Papadopoulos, gr-qc/0101103, to appear in Physics Letters B.

[7] T. Christodoulakis, E. Korfiatis and G.O. Papadopoulos, gr-qc/0107050, to appear in Communications in Mathematical Physics

[8] see e.g. L. D. Landau \& E. M. Lifshitz, "The Classical Theory of Fields", Pergamon Press, (1986).

M. Tsamparlis \& P. S. Apostolopoulos, Journal of Mathematical Physics 41(11) (2000) 7573.

[9] A. Ashtekar and J. Samuel, Classical \& Quantum Gravity 82191 (1991).

V. N. Folomeev and V. Ts. Gurovich, Gravitation \& Cosmology, 6 (2000) No. 1 pp. 19-26.

S. Hervik, Classical \& Quantum Gravity 17 (2000) 2765, gr-qc/0003084

M. J. Duncan and L. G. Jensen, Nuclear Physics B 312 (1989) 662

B. K. Berger, Physical Review D 32 (1985) 2485

B. K. Berger and C. N. Vogeli, Physical Review D 32 (1985) 2477. 
[10] T. Christodoulakis, G. Kofinas, E. Korfiatis, G. O. Papadopoulos and A. Paschos, Journal of Mathematical Physics 42(8) (2001) pp. 3580-3608, gr-qc/0008050.

[11] G. R. F. Ellis \& M. A. H. MacCallum, Communication in Mathematical Physics 12 (1969) 108-141.

[12] G. E. Sneddon, Journal of Physics A 9 (1976) 229;

M. A. H. MacCallum and A. H. T. Taub,

Communications in Mathematical Physics 25 (1972) 173;

T. Christodoulakis and E. Korfiatis, Nuovo Cimento 109 B (1994) 11.

[13] P. A. M. Dirac, Canadian Journal of Mathematics 2 (1950) 129;

Proceedings of Royal Society London A 246 (1958) 333;

Physical Review 114 (1959) 924;

"Lectures on Quantum Mechanics", Belfer Graduate School of Science, Yeshiva University, New York 1964.

[14] K. V. Kuchař and P. Hajiceck, Physical Review D 41 (1990) 1091;

Journal of Mathematical Physics 31 (1990) 1723.

[15] K. V. Kuchař, Journal of Mathematical Physics 23(9) (1982) 1647-1661.

[16] A. Harvey, Journal of Mathematical Physics 20(2) (1979) 251-258.

[17] P.R. Carabedian 'Partial Defferential Equations", Chelsea, New York 1986.

[18] M. Henneaux, M. Pilati \& C. Teitelboim Physics Letters B110 123 (1982) 